\section{Mean Square Radius of randomly coiled Molecular Chains}

AN expression for the mean square radius of a randomly coiled long-chain polymer, that is, for the mean square distance of its constituent groups from their centre of mass, was first derived by Debye ${ }^{1}$. Debye's formula applies only to very long chains; the following simple derivation is valid for any unbranched chain, provided the correlation between two atoms depends only on the number of links separating them (this implies neglect of the excluded volume effect).

According to a theorem by Lagrange ${ }^{2}$, in a system of $n$ particles the mean square distance $R_{n}{ }^{2}$ of the particles from their mass centre is given (in the case of equal masses) by :

$$
R_{n}^{2}=\sum_{i} r_{G i}{ }^{2} / n=\sum_{i, j} r_{i j}{ }^{2} / n^{2}
$$

Here the $r_{G i}$ denote the distances of the individual particles from the mean centre and the $r_{i j}$ their mutual distances; the double sum has to be taken over all pairs of particles (once only). If the particles are arranged in the form of a chain and the average is taken over all possible configurations of the chain, equation (1) leads to :

$$
\begin{array}{r}
n^{2}<R_{n^{2}}>=\Sigma<r_{i j^{2}}>=(n-1)<r_{12}{ }^{2}>+ \\
(n-2)<r_{13}{ }^{2}>+\ldots+<r_{1 n^{2}}>
\end{array}
$$

where the signs $<>$ denote statistical averages.

For links of constant length $l_{0}$ and mutually independent directions :

$$
\left\langle r_{1 j}{ }^{2}\right\rangle=(j-1) l_{0}{ }^{2},
$$

which yields with the use of $(2)$ :

$$
\begin{aligned}
&<R_{n}{ }^{2}>=\frac{1}{6} l_{0}{ }^{2}(n-1 / n)= \\
& \frac{1}{6}<r_{1 n^{2}}>(1+1 / n) .
\end{aligned}
$$

For a constant valency angle $\pi-\omega$ and free rotation about bonds, it is known that ${ }^{3}$ :

$$
<r_{1} j^{2}>=l_{0}^{2}\left[(j-1) \frac{1+\alpha}{1-\alpha}-2 \frac{\alpha-\alpha^{j}}{(1-\alpha)^{2}}\right],
$$

where $\alpha=\cos \omega$. Substitution of this into (2) yields

$$
\begin{aligned}
& <R_{n}{ }^{2}>=\frac{l_{0}{ }^{2}}{n^{2}}\left[\frac{1}{6} \frac{1+\alpha}{1-\alpha}\left(n^{3}-n\right)-\right. \\
& \left.\quad \frac{(n-1) \alpha}{(1-\alpha)^{2}}\left(n-\frac{2 \alpha}{1-\alpha}\right)-\frac{2\left(\alpha^{3}-\alpha^{n+2}\right)}{(1-\alpha)^{4}}\right] .
\end{aligned}
$$

More complicated correlations can be treated in an analogous way. In all cases in which there is no preferential tendency for the chain to develop in any specific direction in space, Debye's result ${ }^{1}$ is obtained for large $n$, that is :

$$
<R_{n}^{2}>=<r_{1 n^{2}}>\left[\frac{1}{6}+O\left(n^{-1}\right)\right] .
$$

The subject of this note formed part of the fundamental research programme of the British Rayon Research Association.

Department of Theoretical Physics,

$$
\text { R. A. SACK }
$$

University of Liverpool. Nov. 24.

\footnotetext{
'Debye, P., J. Chem. Phys., 14, 639 (1946).

"Lagrange, 'J.-L., Oeuvres, 5, 535 (Paris, 1870). Cf. Lamb, H. s Wall, F. T., J. Chem. Phys., 11, 67 (1943).
}

\section{Follicle Development in the Australian Merino}

So far as we are aware, there is no published information of a quantitative nature on the postnatal development of the follicle population in the skin of the merino sheep, although Carter and Hardy ${ }^{1}$ have presented quantitative data for the pre-natal period. In his classical description of the development of the follicle group, Carter ${ }^{2}$ was concerned with descriptive morphology and ontogenic considerations rather than with quantitative aspects. His clear definition of the two follicle types (primary and secondary), their relative place in the developmental sequence, and their fundamental arrangement in group form lays the foundation on which quantitative considerations may be built. The most satisfactory measure of the progress of development of the follicle population is the ratio of secondary follicles to primary follicles $(S / P)$. The validity of this unit rests on the observation (Carter ${ }^{2}$ ) that the primary population within any region is determined by approximately the eighty-sixth day of pre-natal life, and that only secondary follicles develop thereafter. While it has not been proved that no primary follicles develop after this time, the histological evidence is heavily in this direction.

Unpublished observations at this College on causes of variability in the skin and fleece of merino sheep have shown that, at fifteen months of age, the $S / P$ ratio of twins is frequently lower than that of singles, and those for the progeny of young ewes are also lower than those of mature ewes. It was decided, therefore, to study the development in these classes from early post-natal life. Skin sections were taken from the mid-side at $10,52,66,82,92,113,185$ and 426 days of age, a single section of $1 \mathrm{~cm}$. diameter being taken from each animal at each sampling period.

These sections were examined histologically by a method which was essentially the same as that described by Carter $^{3}$. The accompanying graph shows the relationship between the $S / P$ ratio and age in three groups of lambs taken at random from a flock of South Australian (Bungaree) merino.

The consistently lower ratio in those groups subject to some nutritional penalty (twins and progeny of young ewes) is quite evident. Theoretically, it is not possible for the $S / P$ ratio to decline unless there is a loss of secondary follicles. Whether this is the cause of the three points showing a lower ratio than at the previous observation cannot be stated with certainty. Such a decline in ratio could also appear as a result of sampling variations, although this seems unlikely in view of the numbers involved. Of particular interest is the relatively large difference in the

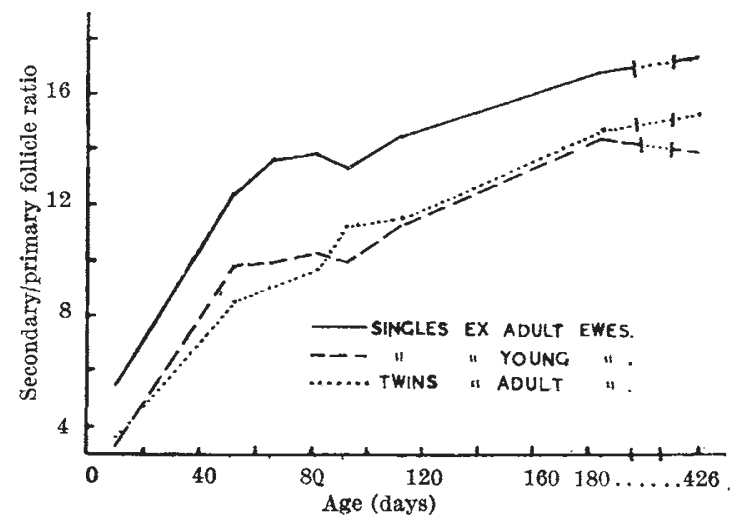

